\title{
Organic solar cells with inverted layer sequence incorporating optical spacers - simulation and experiment.
}

\author{
Birger Zimmermann ${ }^{a}$, Markus Glatthaar ${ }^{a}$, Michael Niggemann Author $3^{a, b}$, Moritz Kilian \\ Riede $^{b}$, Tobias Ziegler ${ }^{b}$, Andreas Gombert ${ }^{b}$ \\ ${ }^{a}$ Freiburg Materials Research Center (FMF), Stefan-Meier-Strasse 21, 79104 Freiburg, \\ Germany; \\ ${ }^{b}$ Fraunhofer Institute for Solar Energy Systems ISE, Heidenhofstrasse 2, 79110 Freiburg, \\ Germany
}

\begin{abstract}
In this paper we present detailed optical simulations of organic bulk-heterojunction solar cells built with inverted layer sequence as compared to the commonly used setup which is based on indium tin oxide (ITO) covered glass or plastic substrates and where the metal electrode is evaporated on top of the active absorber blend. The inverted setup may have production related advantages over the conventional setup, as the metal electrode is first evaporated onto the substrate and afterwards only wet chemical processes are needed. Additionally ITO can be replaced with a suited module concept. The effects of light trapping with an optical spacer, namely a transparent conductive layer between the absorber and the metallic electrode are investigated for the inverted setup. The results show that the insertion of an optical spacer does not increase the maximal obtainable short circuit current density and is only beneficial if a decrease of film thickness of the active absorber results in a higher internal quantum efficiency, open circuit voltage or fill factor. In the experimental section we show that the inversion of the layer sequence can be realised without any loss in device efficiency as compared to devices with the conventional layer sequence.
\end{abstract}

Keywords: organic solar cell, inverted layer sequence, optical simulation, optical spacer

\section{INTRODUCTION}

On the way towards commercialisation of organic bulk-heterojunction solar cells, efficiency, stability and low costs are the three crucial requirements to be met. Efficiencies approaching $5 \%$ have been reported ${ }^{1}$ and also promising long term stability has been shown for poly(3-hexylthiophene):1-(3-Methoxycarbonyl)-Propyl1-1-Phenyl-(6,6) $\mathrm{C}_{61}$ (P3HT:PCBM) based bulk-heterojunction solar cells. The costs can be minimised through reel-to-reel production and the use of simple, compatible coating technologies for the different inorganic and organic layers. In thin film solar cells with film thicknesses below the absorption depth and wavelength of the absorbed light, interference plays an important role for the optical field inside the active region of the solar cell. For different types of thin film solar cells it has been shown ${ }^{1,2}$ that the insertion of an optical spacer with a suited thickness gives a beneficial distribution of the optical field, because the inevitable node at the reflecting metal electrode is shifted away from the active material. This results in a higher and more homogeneous charge carrier generation over the whole thickness of the active layer. Given two thin cells with the same active film thickness, a device with suited optical spacer would show a higher short circuit current density $J_{s c}$ because of the increase of the optical field inside the active layer. Taking into consideration two cells with active film thicknesses sufficient to absorb all of the incoming light in the spectral region of the absorption of the active material, the one with optical spacer can be made thinner. This device should therefore benefit from higher open circuit voltage and fill factor, the short circuit current density being more or less the same for both devices. The increase in open circuit voltage would arise from a higher density of charges generated and therefore higher quasi fermilevel splitting, while a reduced series resistance would lead to a better fill factor. Conventional organic bulk-heterojunction solar cells are fabricated on indium tin oxide (ITO) covered glass which is first coated with

Further author information: (Send correspondence to Birger Zimmermann )

Birger Zimmermann : E-mail: birger.zimmermann@fmf.uni-freiburg.de, Telephone: +49 7612034795

Photonics for Solar Energy Systems, edited by Andreas Gombert, Proc. of SPIE Vol. 6197, 61970G, (2006) - 0277-786X/06/\$15 - doi: 10.1117/12.662530 
poly(3,4-ethylenedioxythiophene):poly(styrenesulfonate) PEDOT:PSS and subsequently with the active blend of regio regular poly(3-hexylthiophene) P3HT and 1-(3-Methoxycarbonyl)-Propyl-1-1-Phenyl-(6,6) $\mathrm{C}_{61}$ (PCBM). Finally, the metal electrode is evaporated on top of the active layer. Therefore in standard bulk-heterojunction solar cells, the optical spacer must be applied onto the active material, which reduces the choice of materials and methods available, because the organic semiconductors should not be affected by the procedure. We suggest therefore to build the bulk-heterojunction solar cell with inverted layer sequence as described in detail elsewhere, ${ }^{3}$ adding an optical spacer between the metal cathode and the active layer. This configuration has the advantage that the metal cathode and the optical spacer are applied onto the substrate before it is coated with the sensitive organic materials. This facilitates the use of a large variety of materials and processes to form the dielectric layer with the needed qualities. This layer has to be optically transparent for the sunlight, a good electron acceptor and a sufficient conductor for electrons. These requirements are met by a high bandgap semiconductor, whose conduction band is close to the lowest unoccupied molecular orbital of PCBM, for example titanium dioxide.

\section{OPTICAL SIMULATION}

The planar organic solar cell, consisting mainly of a substrate carrying a thin film system, has to be described with coherent optics, as the wavelength of the absorbed light of about $300-800 \mathrm{~nm}$ exceeds the film thicknesses of the different layers used in organic solar cells of typically $50-300 \mathrm{~nm}$. The here applied Rigorous Coupled Wave Analysis (RCWA) is especially suited for describing near-field phenomena at corrugated interfaces but can also be used to simulate the optical field in planar thin film systems.

From the spectral absorption in the active layer the theoretically obtainable short circuit current density $J_{s c}$ is calculated by multiplying the spectral absorption with the solar spectrum at each wavelength of the incident light and integrating over the relevant wavelengths $(300-800 \mathrm{~nm})$. This means that an internal quantum efficiency of unity is assumed independently of the device thickness. The optical constants of the absorber have been determined by ellipsometry. ${ }^{4}$ The values for PEDOT:PSS were published elsewhere ${ }^{5}$ and the values for aluminium and titanium dioxide were taken from ${ }^{6}$ respectively.

\subsection{Results}

Figure 1 shows the theoretically obtainable short circuit current density $J_{s c}$ against the thickness of the active P3HT:PCBM absorber layer for different thicknesses of the optical spacer. For solar cells without spacer, a maximum occurs at $\approx 85 \mathrm{~nm}$, resulting in a short circuit current of $11.2 \mathrm{~mA} / \mathrm{cm}^{2}$. Increasing the active layer thickness further results in a decrease of the short circuit current, which is, at a first glance, counter intuitive. This decrease results from an increased absorption in the PEDOT layer due to an unfavourable optical field distribution, as depicted in fig. 2. A minimum is located at $\approx 140 \mathrm{~nm}\left(9.9 \mathrm{~mA} / \mathrm{cm}^{2}\right)$ followed by a second maximum at $\approx 220 \mathrm{~nm}$ with $J_{s c} \approx 12.0 \mathrm{~mA} / \mathrm{cm}^{2}$. Inserting an optical spacer shifts the maxima to lower absorber thicknesses as expected, but the short circuit current density at the maximum also decreases. The consequence is that it is only favourable to insert an optical spacer, if this loss in short circuit current density is overcompensated by a gain in open circuit voltage or fill factor. In in a more detailed view a reduction of loss due to recombination of charge carriers, which could result in a higher $J_{s c}$ for the cell with spacer, despite the lower overall absorption of light for the cell with optical spacer compared to the cell without spacer. The counter intuitive oscillating behaviour of $J_{s c}$ is more closely analysed in figure 2. One can see that at the maxima of $J_{s c}$ constructive interference in the active layer takes place for longer wavelengths, where the photon flux of the sun is high, whereas in the minimum the condition for constructive interference is fulfilled for shorter wavelengths, where only few photons are emitted by the sun (solid line in fig. 2) The oscillating behaviour hence results from the alternation between constructive and destructive interference for the wavelength region, where most of the current is generated. As can be seen from figure 3, the photons are not lost in a parasitic absorbing layer (for example PEDOT:PSS), but are reflected. These interference effects are not intuitive, but well known from e.g. dielectric mirrors or interference filters. 


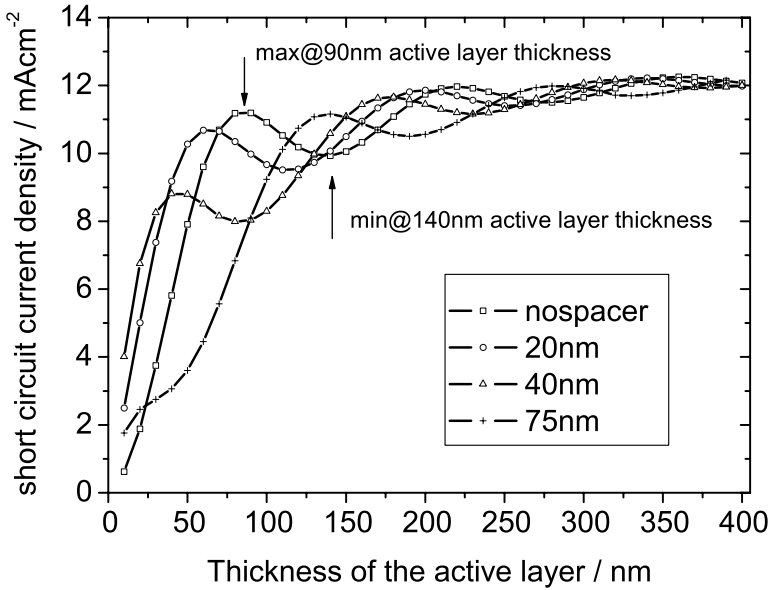

Figure 1. Theoretically obtainable short circuit current for several device thicknesses with different optical spacer thicknesses. Insertion of an optical spacer shifts the local maxima to lower film thicknesses The Arrows indicate first local maximum and first local minimum for the solar cell without spacer, the spectrally resolved absorption in the active layer is depicted in fig. 2 .

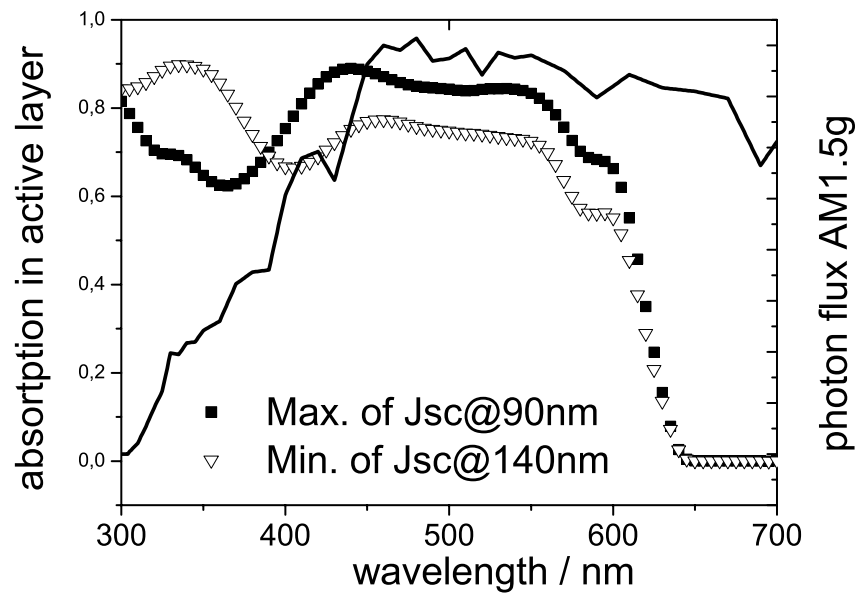

Figure 2. Simulated spectrally resolved absorption in the active layer for the maximum and the minimum of $J_{s c}$ respectively. The photon flux of the AM 1.5 solar spectrum is given for comparison (solid line). If constructive interference takes place for wavelengths more in the red, where the sun irradiates more photons than in the blue region, $J_{s c}$ becomes maximal (arrow "max..." in fig. 1), if constructive interference occurs for wavelengths in the blue region where less photons are delivered by the sun, $J_{s c}$ passes through a local minimum (arrow "min..." in fig. 1). 


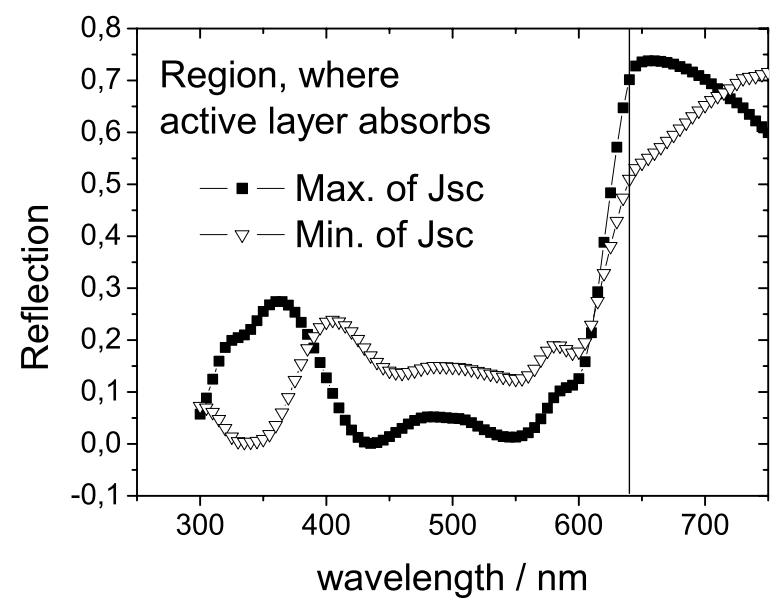

Figure 3. Spectrally resolved reflection for solar cells with $90 \mathrm{~nm}$ (maximum of $J_{s c}$ ) and $140 \mathrm{~nm}$ (minimum of $J_{s c}$ ) active layer thickness. The thicker cell reflects more light due to interference effects, which are commonly utilized e.g. in dielectric mirrors.

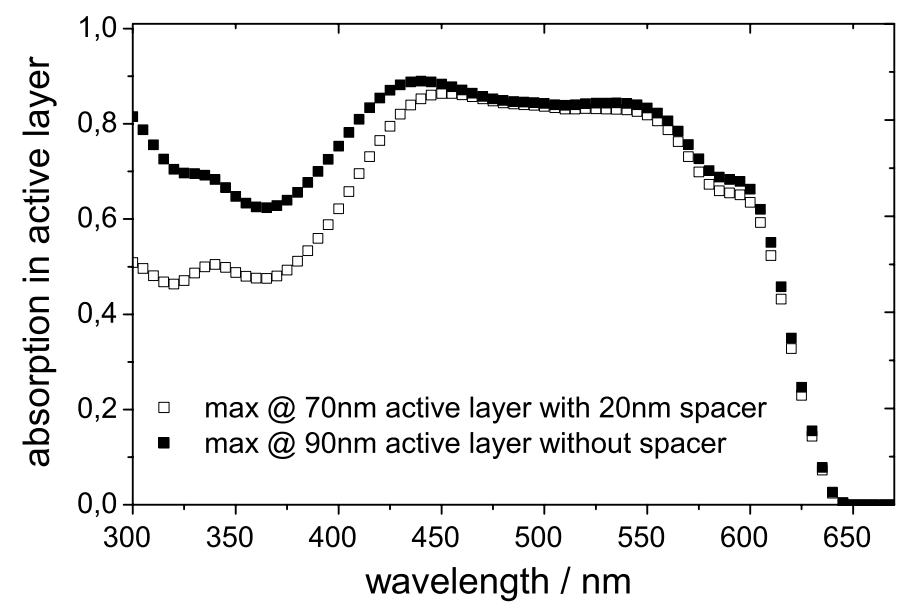

Figure 4. Simulated spectrally resolved absorption in the active layer for the maxima at 90nm active layer thickness for a device without optical spacer and at $70 \mathrm{~nm}$ active layer thickness for a device with $20 \mathrm{~nm}$ thick optical spacer. The effect of the weighting by the solar photon flux is clearly illustrated, as for the device with spacer, a loss in absorption in the blue region can be observed where the photon flux is low, while the two curves nearly coincide in the red region, where the photon flux is high, leading to similar $J_{s c}$ for both devices. 


\section{EXPERIMENT}

Organic solar cells (OSC) with standard and inverted layer sequence have been produced from P3HT:PCBM solution $(26 \mathrm{mg} / \mathrm{ml})$ in a mixing ratio of $6: 5$ in tetrahydronaphtalene as solvent. For standard OSC ITO-covered glass was coated with PEDOT:PSS and subsequently with the active absorber blend. Finally the aluminium electrode has been thermally evaporated. For inverted solar cells first the metallic electrode consisting of aluminium and titanium were evaporated thermally and via electron beam respectively onto a glass plate, followed by the absorber blend and the PEDOT:PSS layer. Finally a metal $(\mathrm{Au})$ grid was evaporated on top of the PEDOT:PSS layer to support the low lateral conductivity of this layer. For comparably prepared samples of both types, we obtain very similar results in power conversion efficiency $\eta$, which amounts to $3 \%$ at 1 sun AM $1.5\left(100 \mathrm{~mW} / \mathrm{cm}^{2}\right)$. The power conversion efficiency was determined on a Steuernagel solar simulator, calibrated with a silicon reference solar cell which has a filtering window to decrease the spectral mismatch between the test cell and the reference cell and using a mismatch factor, which has been determined from the spectral response (SR) of the test cell, the SR of the reference cell, the spectrum of the solar simulator and the solar AM 1.5 spectrum. For details of the mismatch factor method. ${ }^{7,8}$ Figure 5 shows the current voltage characteristics of both cells. The inverted solar cell has a slightly lower $J_{s c}$ because of the shadow loss due to the metal grid, but compensates this by a higher open circuit voltage, resulting in more or less the same power conversion efficiency.

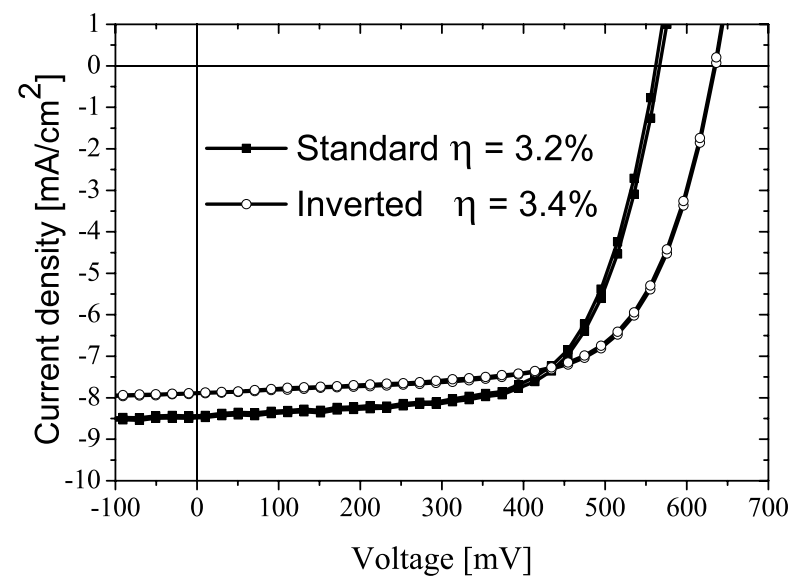

Figure 5. Current voltage curves of standard and inverted organic solar cells illuminated with $100 \mathrm{~mW} / \mathrm{cm}^{2}$ on a solar simulator, corrected for spectral mismatch.

\section{CONCLUSIONS}

The optical simulation of P3HT:PCBM organic bulk-heterojunction solar cells show an oscillating rise of $J_{s c}$ with increasing active layer thickness, leading to local maxima and minima at certain active layer thicknesses. Upon insertion of an optical spacer between the active absorber layer and the metal electrode these extrema are shifted to lower absorber thicknesses, accompanied by a decrease of the $J_{s c}$ value in the respective maximum. A significant overall increase of $J_{s c}$ is only observed for very thin layers, nominally $\leq 70 \mathrm{~nm}$ for P3HT:PCBM based devices. Hence, the insertion of an optical spacer is especially useful for solar cells with strict constraints for the active layer thickness due to a decrease of internal quantum efficiency upon increasing active layer thickness. These conditions are especially fulfilled in small molecule based solar cells, ${ }^{2}$ where the active layer cannot be made sufficiently thick to absorb all of the incoming light. For solar cells with less critical constraints for the active layer thickness, it could be also beneficial to add an optical spacer, if the more homogenous charge carrier generation leads to higher open circuit voltage or fill factor. If the spacer would be much cheaper than the absorber, it may be reasonable to replace not illuminated expensive absorber by a cheap transparent spacer.

Proc. of SPIE Vol. 6197 61970G-5 


\section{REFERENCES}

1. K. Lee, J. Y. Kim, W. Ma, and A. Heeger, "New architectures for thermally stable high efficiency polymer solar cells," Proceedings SPIE optics and photonics San Diego USA 5938, pp. 59380B-1-12, 2005.

2. J. Drechsel, B. Mannig, D. Gebeyehu, M. Pfeiffer, K. Leo, and H. Hoppe, "Mip-type organic solar cells incorporating phthalocyanine/fullerene mixed layers and doped wide-gap transport layers," Organic Electronics 5, pp. 175-186, 2004.

3. M. Glatthaar, M. Niggemann, B. Zimmermann, P. Lewer, M. Riede, A. Hinsch, and J. Luther, "Organic solar cells using inverted layer sequence," Thin Solid Films 491, pp. 298-300, 2005.

4. T. Ziegler, Optische Spektroskopie an organischen Solarzellen, University of Freiburg, 2006.

5. H. Hoppe, N. Arnold, N. S. Sariciftci, and D. Meissner, "Modeling the optical absorption within conjugated polymer/fullerene-based bulk-heterojunction organic solar cells," Solar Energy Materials and Solar Cells 80(1), pp. 105-113, 2003.

6. E. Palik, Handbook of Optical Constants of Solids, Academic Press, 1985.

7. C. H. Seaman, "Calibration of solar cells by the reference cell method-the spectral mismatch problem," Solar Energy 29, pp. 291-298, 1982.

8. K. Bucher, R. Stiening, K. Heidler, K. Emery, H. Field, D. King, and B. Hansen, "Intercomparison of two primary reference cell calibration methods," Proceedings of the 23rd IEEE Photovoltaic Specialists Conference, pp. 1188-1193, 1993. 